\title{
Characterization of humic-like substances of linden pyrochar as stimulators of the intensity of soil respiration
}

Smirnova E.V., Okunev R.V., Giniyatullin K.G., Guseva I.A., Gordeeva K.A. Kazan (Volga region) Federal University, Kazan, Russian Federation, tutinkaz@yandex.ru doi: 10.36291/HIT.2019.smirnova.128

Pyrochar is a promising ameliorant obtained by pyrolysis of organic material under oxygen deficiency conditions, the use of which is aimed at solving two of the most important problems of our time: long-term improvement of soil fertility and the need for atmospheric carbon sequestration [1]. The perspectives for atmospheric carbon dioxide sequestration are associated with the fact that the carbon content in pyrochar, which is usually resistant to oxidation, can be stored in soils from several hundred to thousands of years [2]. Thus, the introduction of pyrochar, prepared and applied to soils in an industrial scale, makes it possible to remove up to $50 \%$ of the carbon of the initial plant residues from the biological circuit [3].

Along with inert components (benzene polycarboxylic acids), a significant part of the carbon in the pyrolysis products of organic materials can be represented by humic substances, glycolipids, phospholipids, etc. [2]. They will be characterized by different resistance to microbial destruction, however, the introduction of these substances with pyrochar can change the availability of available organic food for soil microorganisms. As a result, after the introduction of pyrochar, the $\mathrm{CO}_{2}$ release can increase due to the rapid mineralization of the labile fraction of the pyrogenic material. In our work, using different temperatures of pyrolysis, pyrochars were obtained from the remains of linden wood. Humic-like substances were extracted with an alkaline solution [4]. Their qualitative characteristics were studied by UV spectrometry. To study the effect of humic-like substances on substrate-induced (SIR) and basal respiration of soil, model experiments with the introduction in the soil of native pyrochar and pyrochar treated with an alkaline solution were carried out. The intensity of soil respiration was determined after 3 months and 6 months of incubation.

The results of the study showed that native linden pyrochars added to the soil cause a short-term increase in soil SIR. The addition of pyrochar to the soil modified by treatment with an alkaline solution leads to an additional increase in the emission of $\mathrm{CO}_{2}$ from the soil, which decreases slightly over time.

Acknowledgements. This work was supported in part by the Russian Foundation for Basic Research, research project №17-04-00869.

\section{References}

1. Lehmann J., Joseph S. Biochar for Environmental Management: Science and Technology. London: Earthscan Publ., 2009. P.1-12.

2. Kuzyakov Y. et al. // Soil Biol. Biochem. 2014. 70:229-236.

3. Lehmann J. et al. // Mitig. Adapt. Strat. Glob. Chang. 2006. 11:395-419.

4. Kononova M.M., Belchikova N.P. // Sov. Soil Sci. (Pochvovedenie). 1961. 10:75-87. 